\title{
The use of ROTEM in liver transplant surgeries: a luxurious option
} or a necessity?!

\author{
Muntadhar Al Moosawi ${ }^{1}$ \\ ${ }^{1}$ Affiliation not available
}

February 15, 2022

In 2017, the hepatobiliary surgery team at the Royal Hospital in Oman performed the first successful liver transplant surgery. This medical achievement was the first of a sequence of multiple successful liver transplant surgeries performed in the country. Blood management in liver transplant surgeries can be challenging. In this article I will address some of the strenuous challenges related to blood management in this patient population and discuss the use of rotational thromboelastometry (ROTEM) to mitigate these challenges.

It is well known that patients with liver disease have a rebalanced hemostasis due to the impaired synthesis of both the coagulation factors and pro-coagulant factors. Interestingly, some studies demonstrated an increased endogenous thrombin potential (ETP) in patients with liver cirrhosis ${ }^{1}$. Hence, it is now well known that this group of patients requires special attention when it comes to blood management.

For many years, international normalized ratio (INR) has been used by clinicians to evaluate the hemostatic activity, or as a predictor of bleeding in patients with liver disease. INR is one of the commonly used conventional coagulation assay that has been developed to standardize warfarin monitoring across laboratories. This simple test has many limitations that make it an extremely poor predictor of bleeding in this group of patients. For instance, INR is designed to test the extrinsic and common coagulation pathway factors (i.e Factor VII, X, V, II, and fibrinogen). INR does not take into account the effect of the endogenous anticoagulants such as protein $\mathrm{C}$ and protein $\mathrm{S}$ which are also reduced in patients with liver disease. Therefore, INR provide only limited information about the hemostatic activity ${ }^{2}$.

The practise of transfusing plasma to correct (normalize) INR prior to invasive procedure in liver disease patients is an exercise of futility. In patients with minimally elevated INR $(1.1-1.8)$, transfusing plasma to correct INR resulted in median change of INR of 0.07 only. A significant change in INR was only observed when INR is above $5^{3,4}$. In their most recent guidelines, the society of interventional radiology recommended that INR is not applicable in patients with liver disease who are attending low risk interventional radiology (IR) procedure. The society also recommended that INR of $<2.5$ is acceptable for liver disease patients attending high risk IR procedures ${ }^{5}$.

Patients undergoing liver transplant surgeries are exposed to an incredibly challenging hemostatic environment and blood management in these patients is crucial. Depending on conventional coagulation testing (INR and PTT) to guide blood management in these patients results in an inappropriate transfusion of red cells and plasma and therefore increasing the risk of adverse transfusion reactions as well as volume overload $^{6}$. One of the perhaps not widely known fact about blood transfusion is that the cost of delivering a unit of red cells to a patient can range from 522 to 1183 US dollars, and the cost of delivering a unit of plasma is approximately 409 US dollars ${ }^{7}$.

Newer technologies like ROTEM have been proven to be associated with significantly reduced amount of blood product transfusion in bleeding patients. Thromboelastography (TEG) is another older viscoelastic testing that can also be used in this population. However, compared to TEG, ROTEM provides additional 
information about the entire hemostatic activity ${ }^{8}$. ROTEM is a point of care viscoelastic testing that is performed on whole blood samples collected in trisodium citrate tube (the regular blue top coagulation tube). There are several assays that can be performed on ROTEM, such as EXTEM and FIBTEM. ROTEM results are demonstrated in graph that shows both the coagulation process and fibrinolytic activity. The graph comprises of different parameters as shown in the figure 1. The shape of ROTEM graph can indicate several coagulation abnormalities. Therefore, an appropriate blood product can be transfused accordingly.

There is tremendous evidence to support the use of ROTEM-based algorithms (flow charts) to guide transfusion management. Most of the literature in this field comes from cardiothoracic surgeries, but liver transplant surgeries, trauma and obstetrics are other fields in which ROTEM-guided blood management was shown to be effective. In liver transplant surgeries, the use of ROTEM was associated with $39 \%$ reduction in plasma transfusion in patients with mildly elevated INR (1.1-1.8). The median number of plasma units transfused in liver transplant surgeries reduced significantly from 6 units pre-ROTEM to 2 units post-ROTEM. This significant reduction was also observed with red cell transfusion ${ }^{9}$.

ROTEM also has other advantages. It has a fast turnaround time as it takes approximately 15 minutes for the results to be ready. Once the test is started, the results are demonstrated in the form of ROTEM graph immediately. Also, the results can be accessed remotely. If the test is performed in the laboratory, the results can be viewed in any regular screen in the operation room or anywhere else using a special ROTEM portal that is accessed through a password-protected link.

The use of ROTEM-guided blood management is also cost effective. Although ROTEM test may be slightly more expensive than performing a coagulation profile, the associated reduction in plasma and red cell transfusion counterbalances the testing cost. Leahy MF et al., reported saving of approximately 18 million dollars between 2008 and 2014 after the implementation of ROTEM in their patient blood management ${ }^{10}$.

In conclusion, liver transplant surgeries require unique patient blood management strategy. The use of conventional coagulation testing in these surgeries results in an inappropriate and overutilization of blood products. ROTEM-based algorithms are proven to be associated with reduced red cell and plasma transfusion. ROTEM is an essential component of any liver transplant program. The first stages to implement this technology can be challenging, but with a collaborative effect, the final result will be astonishing.

\section{References}

1. Lisman T, Hernandez-Gea V, Magnusson M, Roberts L, Stanworth S, Thachil J, et al. The concept of rebalanced hemostasis in patients with liver disease: Communication from the ISTH SSC working group on hemostatic management of patients with liver disease. J Thromb Haemost. 2021;19(4):1116-22.

2. Lisman T, Porte RJ. Value of preoperative hemostasis testing in patients with liver disease for perioperative hemostatic management. Anesthesiology. 2017;126(2):338-44.

3. Abdel-Wahab OI, Healy B, Dzik WH. Effect of fresh-frozen plasma transfusion on prothrombin time and bleeding in patients with mild coagulation abnormalities. Transfusion. 2006;46(8):1279-85.

4. Warner MA, Hanson AC, Weister TJ, Higgins AA, Madde NR, Schroeder DR, et al. Changes in international normalized ratios after plasma transfusion of varying doses in unique clinical environments. Anesth Analg. 2018;127(2):349-57.

5. Patel IJ, Rahim S, Davidson JC, Hanks SE, Tam AL, Walker TG, et al. Society of interventional radiology consensus guidelines for the periprocedural management of thrombotic and bleeding risk in patients undergoing percutaneous image-guided interventions-part II: Recommendations: Endorsed by the Canadian association for interventional radiology and the cardiovascular and interventional radiological society of Europe. J Vasc Interv Radiol. 2019;30(8):1168-1184.e1.

6. Fahrendorff M, Oliveri RS, Johansson PI. The use of viscoelastic haemostatic assays in goal-directing treatment with allogeneic blood products - A systematic review and meta-analysis. Scand J Trauma Resusc Emerg Med. 2017;25(1):39. 
7. Callum JL. Bloody easy 4: blood transfusions, blood alternatives and transfusion reactions: a guide to transfusion medicine. Toronto: ORBCoN, Ontario Regional Blood Coordinating Network; 2016.

8. Görlinger K, Pérez-Ferrer A, Dirkmann D, Saner F, Maegele M, Calatayud ÁAP, et al. The role of evidence-based algorithms for rotational thromboelastometry-guided bleeding management. Korean J Anesthesiol. 2019;72(4):297-322.

9. Al Moosawi M, Trudeau J, Smith T, Lefebvre A, Shih AW. ROTEM in the setting of liver transplant surgery reduces frozen plasma transfusion. Transfus Apher Sci. 2021;(103125):103125.

10. Leahy MF, Hofmann A, Towler S, Trentino KM, Burrows SA, Swain SG, et al. Improved outcomes and reduced costs associated with a health-system-wide patient blood management program: a retrospective observational study in four major adult tertiary-care hospitals. Transfusion. 2017;57(6):1347-58.

Figure 1 . Illustration of the graphical representation of ROTEM results with comparison with TEG.

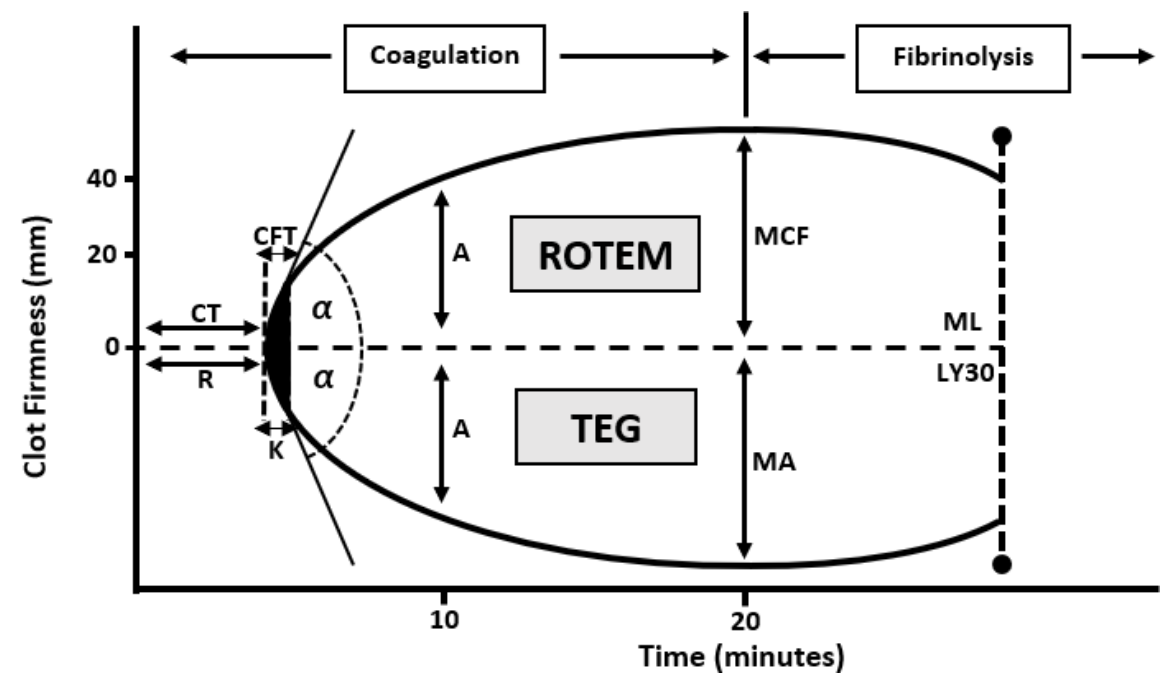

